\title{
Exploring the Relationship between Statins and Alzheimer's Disease: Can Statins Really Prevent Alzheimer's Disease?
}

\author{
Massud Atta \\ College of Medicine, American University of Antigua, St. John's, Antigua and Barbuda \\ Email: massud.atta@gmail.com
}

Received 22 January 2015; accepted 16 February 2015; published 17 February 2015

Copyright (C) 2015 by author and Scientific Research Publishing Inc.

This work is licensed under the Creative Commons Attribution International License (CC BY). http://creativecommons.org/licenses/by/4.0/

c) (i) Open Access

\begin{abstract}
Alzheimer's Disease is a common form of dementia that has neurological and behavioral repercussions. It is definitively diagnosed usually via an autopsy when the following three common markers are found: flattened cortical gray matter, Tau proteins and Beta-amyloid proteins. Patients suffering from Down Syndrome or Parkinson's Disease are often associated with Alzheimer's because they have 2 of the 3 precursors. There has been extensive research on Alzheimer's and many studies claim that statins, a cholesterol-reducing drug, can help prevent Alzheimer's Disease resulting in low levels of beta-amyloid. However, there are several reasons to believe that this is not true and in some cases statins may even induce AD. This paper will examine the pathology of Alzheimer's, the effect of statins and whether it truly plays a role in preventing AD.
\end{abstract}

\section{Keywords}

Statins, Alzheimer's Disease, Cholesterol, Dementia

\section{Background \& Hypothesis}

Alzheimer's Disease is a neurological disorder causing memory loss as well as behavioral and thinking changes. It is the most common cause of dementia and the prevalence is increasing among geriatric populations. Some studies show that statins (a family of cholesterol lowering drugs) can help prevent or lower the risk of Alzheimer's; however, other studies show that there are no correlations between statins and Alzheimer's or statins perhaps may cause an increasing risk of Alzheimer's. The intention of this paper is to research this idea, hypothesizing that statins will have an increased risk of Alzheimer's disease. 


\section{Introduction}

Alzheimer's Disease is a rapidly growing neurological issue in the United States. About 2.3 million people suffered from AD in 1997, a majority of whom were women [1]. According to "alz.org", the current number of patients living with Alzheimer's disease has nearly doubled (to 5 million) since 1997, costing 15.4 million careproviders \$216 billion dollars of "unpaid care" [2]. In other words, it is a very dangerous and costly disease. Alzheimer's disease is the most common form of dementia, which is a common term for decreased mental ability often affecting everyday activities.

\section{Methods}

This research is purely based on literature from previous studies as well as lectures in the field of neurology.

\section{Etiology and Diagnosis of Alzheimer's Disease}

The only correlation causing Alzheimer's disease seen has been age. The increasing age of individuals have higher risk of developing AD than younger populations. With this, Alzheimer's is technically not diagnosed until autopsies are completed where a decrease in synthesis of acetylcholine is seen from the Nucleus Basalis [3]. In addition, neurologists search for Plaques (beta-amyloid proteins) and Tangles (Tau proteins). Beta-amyloids build up between nerve cells and Tau proteins develop inside cells, both of which slow the neurotransmission in the brain. Since the early 1990s, beta-amyloids have been especially linked to Alzheimer's disease since the gene for Amyloid Precursor Protein (APP) is found on chromosome 21 [4]. Interestingly, in Trisomy 21 or Down Syndrome a duplicate copy of this gene is made and patients with Down Syndrome almost inevitably develop AD as early as 40 years old. According to current neurologists, Down Syndrome and Parkinson's Disease are precursors to Alzheimer's Disease because 2 of the 3 precursors of AD (flattened cortical gray portion of brain, beta-amyloids, Tau proteins) are seen in these disorders [3].

However, there have been increasing studies that link high levels of cholesterol that lead to the development to Alzheimer's disease. For example, the research review article, "Relation between Cholesterol Levels, Statins and Alzheimer's Disease in the Human Population", has found that decreasing plasma cholesterol levels has been associated with a 70\% decrease risk of developing Alzheimer's disease [5]. However, the same article admits that further research needs to be done to know whether the relationship is 'direct, causative or indirect' because it is known that not all statins have the same effect to prevent AD.

\section{Current Treatment and Prevention Options for Alzheimer's Disease}

Currently there is no treatment for Alzheimer's disease and cannot be reversed. However, there are medications that target symptoms of AD and help maintain cognitive function or behavior. Acetylcholinesterase is an enzyme that destroys acetylcholine, a neurotransmitter that is used in synaptic signaling. This enzyme is also the target of many drugs such as Donepezil, Rivastigamine and Galantamine are used to treat the symptoms of AD [6]. The goal of these drugs is to prolong the presence of the acetylcholine in the synapse between neurons to perpetuate signaling in brain synapses.

Another interesting method for treating Alzheimer's disease is anti-beta amyloid protein anti-bodies. The Alzheimer's Disease pathogenesis usually involves the presence of mis-folded beta-amyloid proteins (Tau proteins). If the brain does not clear the proteins properly, neurological problems will arise. A study conducted by W. Guo, S. Sha, T. Jiang, X. Xing and Y. Cao has shown that introducing a plasmid that can be transcribed and translated into an antibody targeting these proteins may help the progression of $\mathrm{AD}$ [7]. In theory, the anti-bodies can be used to clear the proteins in the central nervous system.

According to recent literature, there are methods of preventing of Alzheimer's disease. Research conducted by Complutense University in Madrid, Spain discovered that cannabinoids help prevent beta-amyloid plaques by decreasing microglial activation [8]. Cannabinoid receptors (CB1) are found throughout the nervous system and activation of these receptors decreases microglial activity; specifically decreases astrocyte reuptake of glutamate (an excitatory neurotransmitter).

In addition, a study published in The Official Journal of the American Academy of Neurology claims there is a strong correlation of physical activity and mental decline leading to Alzheimer's disease. The study kept track of 716 geriatric subjects and monitored their exercise habits and well as their cognitive function. It was discov- 
ered that the more daily physical activity decreases the risk of developing Alzheimer's disease [9].

However, another type of treatment is growing in popularity claims to decrease the risk of AD. A family of drugs, called statins, has been recognized to help prevent Alzheimer's because it lowers cholesterol. When there are high circulating levels of cholesterol, an increase level of beta-amyloids can develop eventually leading to Alzheimer's Disease [10]. However, further discussion of this will reveal that this may not be the case.

\section{Cholesterol Synthesis and Statins}

In order to understand whether or not statins can reduce the risk of Alzheimer's disease, the mechanism of statins should be reviewed. Cholesterol is synthesized in the liver through a complex pathway. Acetyl-CoA, a product of beta-oxidation is combined with Acetoacetyl CoA to make HMG-CoA, a precursor to cholesterol biosynthesis. Once formed, HMG-CoA is converted to Mevalonate by the enzyme HMG-CoA Reductase. Mevalonate is further reduced to through a series of reactions to make cholesterol. Statins are a family of drugs that target HMG-CoA Reductase. These drugs mimic HMG-CoA and act as a competitive inhibitor thereby lowering the activity of HMG-CoA Reductase. Statins are typically given to patients with cardiovascular deficits because of its anti-atherosclerotic effects [11]. In addition, statins are known to decrease levels of LDL cholesterol, which are known to fats and cholesterol to extrahepatic tissue.

\section{Can Statins Really Prevent Alzheimer's?}

The argument against statins preventing Alzheimer's can first be simply looked in the statistics of each in the United States. According to a Harvard study, it was recorded that between the ages of $45-64,3 \%$ of men in the study and 2\% of women were taking statin drugs between 1988 and 1994. Between 2005 and 2008, 18\% of men in the same age group were taking statins and $15 \%$ of women in the study were taking the drugs [12]. The percentage of men and women over 65 years of age projected even higher percentages meaning that the rise of patients taking statins is continuously increasing. Logically speaking, if there is a rise in statin use, and statins presumably decreases the risk of Alzheimer's disease, then there should be an inverse relationship in statistics. But according to the Alzheimer's Association, there is an exponential increase in the prevalence of Alzheimer's. Between 2000 and 2010, the number Alzheimer's related deaths has increased $68 \%$ and it is projected that the current 5.2 million Alzheimer's sufferers will rise $40 \%$ by 2025 [13]. So what do all these statistics mean? The rise in statin drug users should have a decrease or an inverse effect on the number of Alzheimer's patients; however, the statistics show that both have significantly increased throughout the past 2 decades. Even in the study The Effect of HMG-CoA Reductase Inhibitors on Cognition in Patients With Alzheimer's Dementia: A Prospective Withdrawal and Rechallenge Pilot Study mentions: "With aging of the U.S. population and the high prevalence of cardiovascular disease and dementia in this population, increasing numbers of older people, including those with dementia, are being treated with statins" [14]. This is just a simple statistical paradigm of how statins are not good preventative drug for Alzheimer's Disease.

Again, the idea behind some research that statins play a role to lower the risk of developing Alzheimer's is due to the idea that increasing levels of cholesterol in the brain will lead to beta-amyloids; as already discussed, beta-amyloids are a precursor to Alzheimer's. However, not all statins that are distributed widely today are able to pass the blood-brain barrier to take effect on cholesterol levels that develop into beta-amyloids. In fact, drugs such as pravastatin have very little lipophilicity, which essentially has very little effect on brain cholesterol levels at all [15].

It is implied that if statins can decrease the risk of Alzheimer's (or decrease AD presence), then it should also be thought that high levels of cholesterol (hyper cholesterolemia) could eventually lead to AD. In a study by Miia Kivipelto, high serum cholesterol levels $(>6.5 \mathrm{mmol} / \mathrm{L})$ in midlife results in high risk of developing Alzheimer's Disease [16]. With this conception, people suffering from AD should also have increased levels of cholesterol circulating in the blood and brain [17]. However, there has been little to no research that comments on the hypercholesterolemia in patients with Alzheimer's disease. In addition, hypercholesterolemia is followed by cardiovascular problems such as Coronary Artery Disease and atherosclerosis, which are not always seen in Alzheimer's patients.

As mentioned before, cholesterol levels in AD patients would (hypothetically) be increased if statins could prevent the disease. But according to a recent study, Alzheimer's is caused by a lower intake in omega- 3 fatty acids, found in fish [18]. The study further explains that cholesterol esters are a common pool for fatty acids in 
plasma. In fact, it is found that there are significantly lower numbers of fatty acids, phospholipids and esterified cholesterol in the CSF of confirmed Alzheimer's subjects [19]. This contradicts the idea that Alzheimer's develops from hypercholesterolemia and the idea that statins can play a preventative role in AD.

Physiologically speaking, myelin sheaths that wrap around and insulate axons are composed of fatty membranes and cholesterol. So it would be important to have a healthy amount of cholesterol and fats circulating the central nervous system (CNS). In theory, myelin sheaths of the CNS, called oligodendrocytes, help to insulate and increase the speed at which action potentials may propagate faster throughout the axon. From node to node, the action potentials transmit signals with the help of fatty myelin sheaths. However, if we decrease the amount of fatty acids and cholesterol to the point where myelin are not able to form, the CNS functionality will likely suffer. Dr. Stephanie Seneff also brought up this idea in 2009 with her article, "APOE-4: The Clue to Why Low Fat Diets and Statins may Cause Alzheimer's". Dr. Seneff also claims that lower levels of fatty acids are found in the CSF in patients with Alzheimer's. In fact, it was found that about Alzheimer's patients have less that $20 \%$ of the concentration of fatty acids in CSF compared to non-Alzheimer's patients [20]. Considering that statins can further reduce this number by lower the carry capacity of fats by cholesterol, it should be made aware that statins can possibly pose a threat to provoking Alzheimer's rather than preventing it.

\section{Conclusion}

It has been proven from many research sources that statins are not very good at preventing Alzheimer's Disease. In fact, there is very little correlation between statins and decreasing levels of beta-amyloid proteins, a marker used to diagnose AD. Some argue that statins may even cause a statin-induced dementia because axons are covered by fatty membranes and help with cognitive activity. Statins, especially ones that cross the blood-brain barrier, can do harm to axons by lowering the circulating levels of fatty acids and cholesterol, which provide insulation of axons. In addition, the statistical growing number of statin users and the rise in prevalence of Alzheimer's contradict the theory that statins decrease Alzheimer's. It appears that statins have very little effect on preventing Alzheimer's and there is a definite possibility that it may even induce AD. This will play a significant role in patients taking their medications. Statins are a highly distributed drug in today's pharmaceutical market, and the decrease in the usage among cardiovascular patients due to the fear of developing Alzheimer's may have a negative impact. However, this may also enable patients suffering from hypercholesterolemia to make lifestyle changes to avoid taking statin drugs.

\section{References}

[1] Brookmeyer, R., Gray, S. and Kawas, C. (1998) Projections of Alzheimer's Disease in the United States and the Public Health Impact of Delaying Onset. American Journal of Public Health, 88, 1337-1342. http://dx.doi.org/10.2105/AJPH.88.9.1337

[2] What Is Alzheimer's? http://www.alz.org/alzheimers_disease_what_is_alzheimers.asp

[3] Glasser, S. (2013) Alzheimers. Neurology Course Lecture. American University of Antigua. Antigua, St. Johns.

[4] Hardy, J. and Allsop, D. (1991) Amyloid Deposition as the Central Event in the Aetiology of Alzheimer's Disease. Trends in Pharmacological Sciences, 12, 383-388.

[5] Austen, B., Christodoulou, G. and Terry, J.E. (2002) Relation between Cholesterol Levels, Statins and Alzheimer's Disease in the Human Population. Journal of Nutrition, Health \& Aging, 6, 377-382. http://www.ncbi.nlm.nih.gov/pubmed/12459888

[6] About Alzheimer's Disease: Treatment. National Institute on Aging.

[7] Guo, W.S., Jiang, T.Z., Xing, X.N. and Cao, Y.P. (2013) A New DNA Vaccine Fused with the C3d-p28 Induces a Th2 Immune Response against Amyloid-Beta. Neural Regeneration Research, 8, 2581-2590.

[8] Ramirez, B., Blazquez, C., et al. (2005) Prevention of Alzheimer's Disease Pathology by Cannabinoids: Neuroprotection Mediated by Blockade of Microglial Activation. Journal of Neuroscience, 25, 1904-1913.

[9] Buchman, A.S., Boyle, P.A., et al. (2012) Total Daily Physical Activity and the Risk of AD and Cognitive Decline in Older Adults. Official Journal of the American Academy of Neurology, 78, 1323-1329.

[10] Fonseca, A.C., Resende, R., Catarina, O. and Pereira, C. (2010) Cholesterol and Statins in Alzheimer's Disease: Current Controversies. Experimental Neurology, 223, 282-293.

http://www.sciencedirect.com/science/article/pii/S0014488609003872

[11] Stancu, C. and Sima, A. (2001) Statins: Mechanism of Action and Effects. Journal of Cellular and Molecular Medicine, 
5, 378-387. http://www.ncbi.nlm.nih.gov/pubmed/12067471 http://dx.doi.org/10.1111/j.1582-4934.2001.tb00172.x

[12] Wehrwein, P. (2011) Statin Use Is up, Cholesterol Levels Are down: Are Americans' Hearts Benefiting? Harvard Health Publications.

http://www.health.harvard.edu/blog/statin-use-is-up-cholesterol-levels-are-down-are-americans-hearts-benefiting-2011 $\underline{04151518}$

[13] Alzheimer's Facts and Figures. Alzheimer's Association. http://www.alz.org/alzheimers_disease_facts_and figures.asp

[14] Padala, K., Padala, P., McNeilly, D., Geske, J., Sullivan, D. and Potter, J. (2012) The Effect of HMG-CoA Reductase Inhibitors on Cognition in Patients with Alzheimer's Dementia: A Prospective Withdrawal and Rechallenge Pilot Study. The American Journal of Geriatric Pharmacotherapy, 10, 296-302. http://dx.doi.org/10.1016/j.amjopharm.2012.08.002

[15] Wagstaff, L., Mitton, M., Arvik, B.M. and Doraiswamy, P.M. (2003) Statin-Associated Memory Loss: Analysis of 60 Case Reports and Review of the Literature. Pharmacotherapy, 23, 871-880.

[16] Kivipelto, M., Helkala, E.L., Laasko, M.P., Haaninien, T., Alhainien, K., Soinenien, H., Tuomilehto, J. and Nissinen, A. (2001) Midlife Vascular Risk Factors and Alzheimer's Disease in Later Life: Longitudinal, Population Based Study. British Medical Journal, 322, 1447-1451. http://www.bmj.com/content/322/7300/1447.long

[17] Wood, W.G., Igbavboa, U., Eckert, G.P., Johnson-Anuna, L.N. and Müller, W.E. (2005) Is Hypercholesterolemia a Risk Factor for Alzheimer's Disease? Molecular Neurobiology, 31, 185-192.

[18] Cunnane, S.C., Schneider, J.A., Tangney, C., Tremblay-Mercier, J., Fortier, M., Bennett, D.A. and Morris, M.C. (2012) Plasma and Brain Fatty Acid Profiles in Mild Cognitive Impairment and Alzheimer's Disease. Journal of Alzheimer's Disease, 29, 691-697.

[19] Mulder, M., Ravid, R., Swaab, D.F., de Kloet, E.R., Haasdijk, E.D., Julk, J., van der Boom, J.J. and Havekes, L.M. (1998) Reduced Levels of Cholesterol, Phospholipids, and Fatty Acids in Cerebrospinal Fluid of Alzheimer Disease Patients Are Not Related to Apolipoprotein E4. Alzheimer Disease and Associated Disorders, 12, 198-203. http://www.ncbi.nlm.nih.gov/pubmed/9772023

[20] Seneff, S. (2009) APOE-4: The Clue to Why Low Fat Diet and Statins May Cause Alzheimer's. http://people.csail.mit.edu/seneff/alzheimers_statins.html 
Scientific Research Publishing (SCIRP) is one of the largest Open Access journal publishers. It is currently publishing more than 200 open access, online, peer-reviewed journals covering a wide range of academic disciplines. SCIRP serves the worldwide academic communities and contributes to the progress and application of science with its publication.

Other selected journals from SCIRP are listed as below. Submit your manuscript to us via either submit@scirp.org or Online Submission Portal.
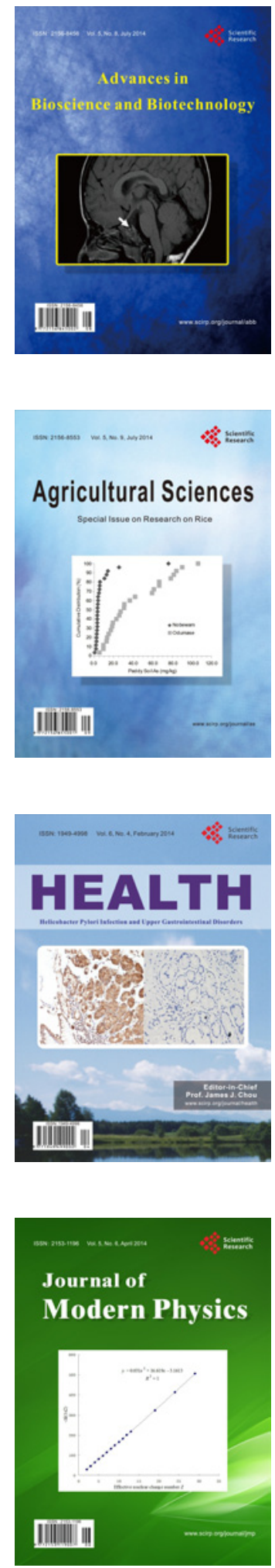
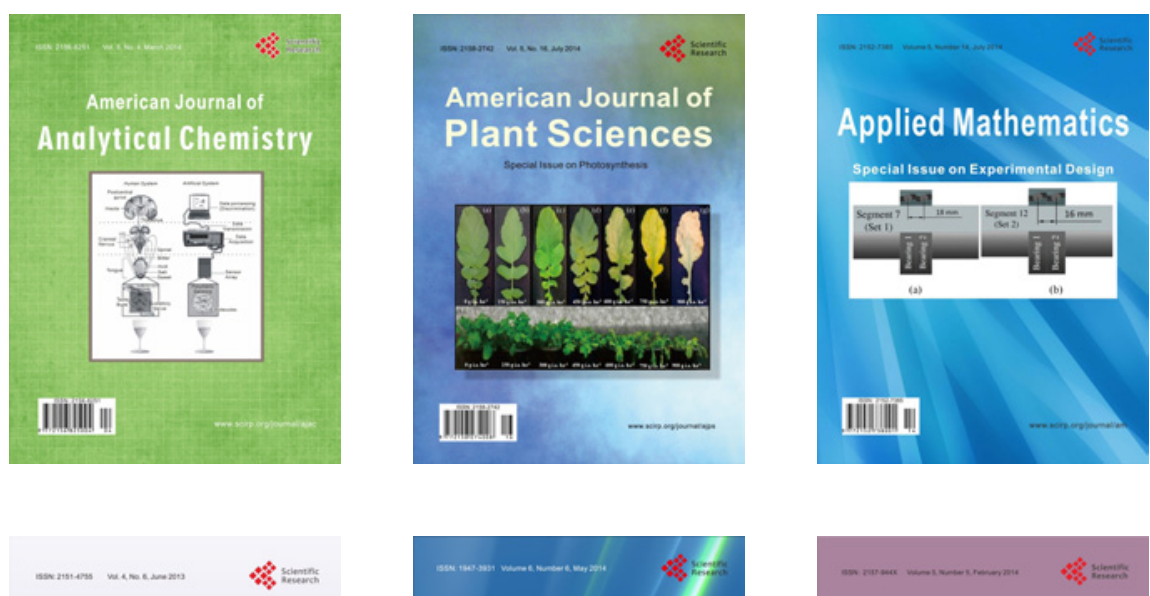

Creative Education
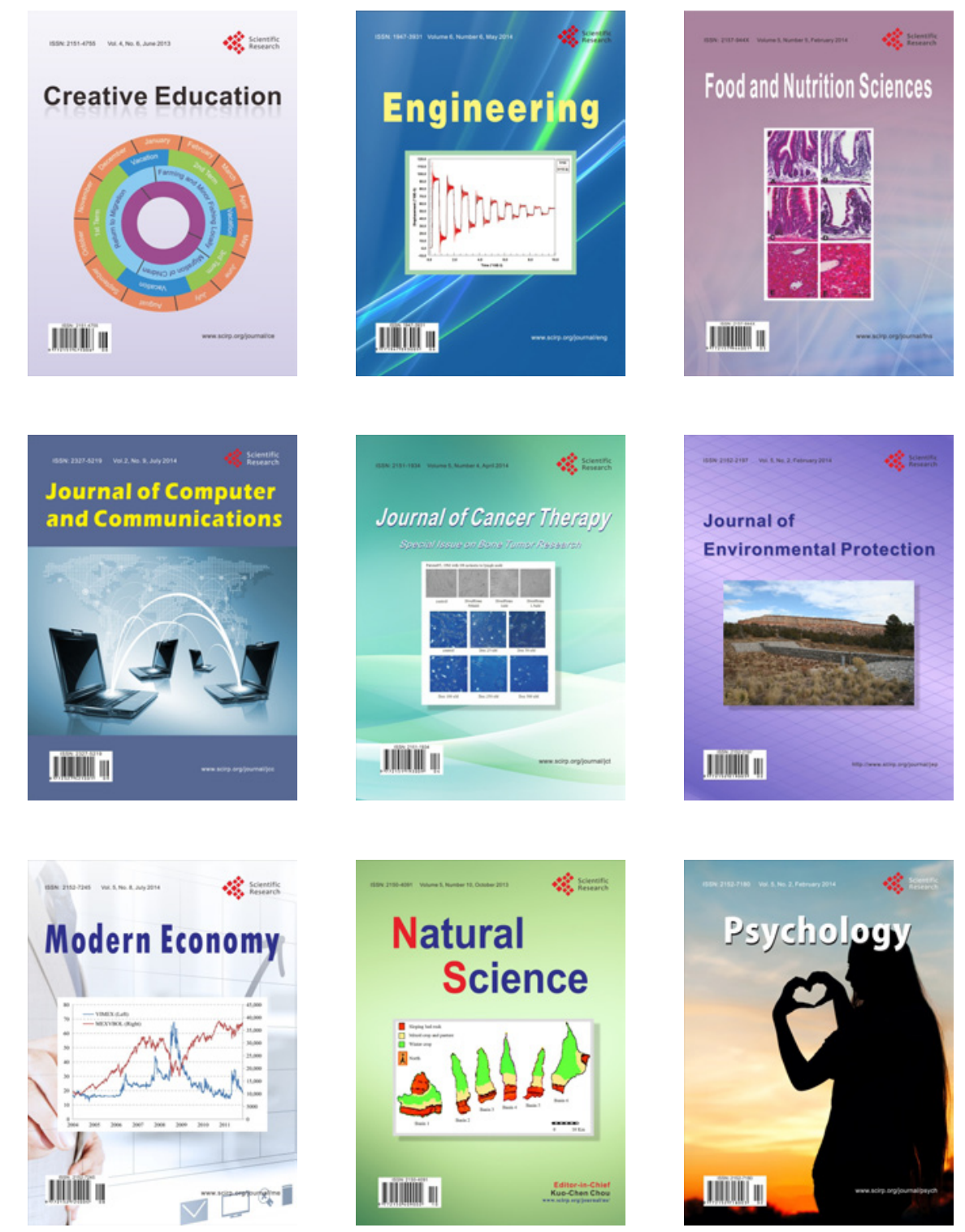